Braz J Med Biol Res, January 2012, Volume 45(1) 86-92

doi: 10.1590/S0100-879X2011007500152

MMP-1/PAR-1 signal transduction axis and its prognostic impact in esophageal squamous cell carcinoma

Hong-hua Peng, Xi Zhang and Pei-guo Cao

The Brazilian Journal of Medical and Biological Research is partially financed by
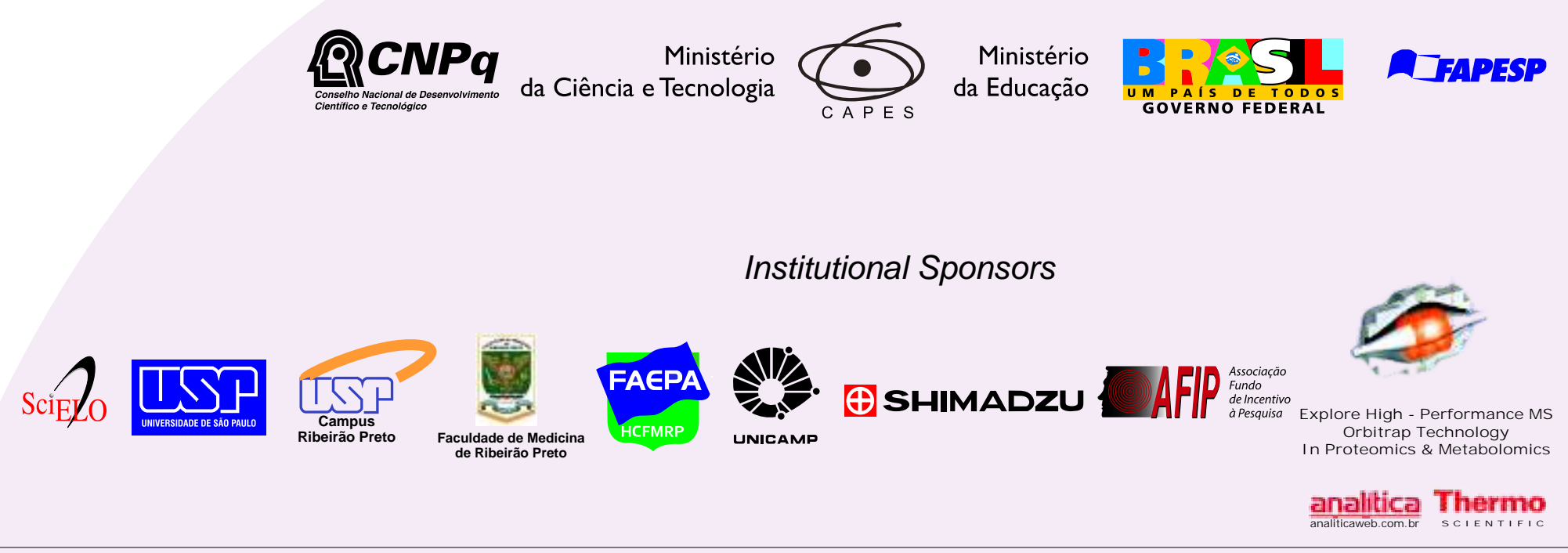


\title{
MMP-1/PAR-1 signal transduction axis and its prognostic impact in esophageal squamous cell carcinoma
}

\author{
Hong-hua Peng, Xi Zhang and Pei-guo Cao \\ Department of Oncology, the Third Xiangya Hospital, Central South University, Changsha, \\ Hunan Province, China
}

\begin{abstract}
The matrix metalloprotease-1 (MMP-1)/protease-activated receptor-1 (PAR-1) signal transduction axis plays an important role in tumorigenesis. To explore the expression and prognostic value of MMP-1 and PAR-1 in esophageal squamous cell carcinoma (ESCC), we evaluated the expression of two proteins in resected specimens from 85 patients with ESCC by immunohistochemistry. Sixty-two (72.9\%) and $58(68.2 \%)$ tumors were MMP-1- and PAR-1-positive, respectively, while no significant staining was observed in normal esophageal squamous epithelium. MMP-1 and PAR-1 overexpression was significantly associated with tumor node metastasis (TNM) stage and regional lymph node involvement. Patients with MMP-1- and PAR-1-positive tumors, respectively, had poorer disease-free survival (DFS) than those with negative ESCC $(P=0.002$ and 0.003 , respectively). Univariate analysis showed a significant relationship between TNM stage [hazard ratio $(\mathrm{HR})=2.836,95 \%$ confidence interval $(\mathrm{Cl})=1.866-4.308]$, regional lymph node involvement $(\mathrm{HR}=2.955,95 \% \mathrm{Cl}=1.713-5.068), \mathrm{MMP}-1$ expression $(\mathrm{HR}=2.669$, $95 \% \mathrm{Cl}=1.229-6.127)$, and PAR-1 expression $(\mathrm{HR}=1.762,95 \% \mathrm{Cl}=1.156-2.883)$ and $\mathrm{DFS}$. Multivariate analysis including the above four parameters identified TNM stage $(\mathrm{HR}=2.035,95 \% \mathrm{Cl}=1.167-3.681)$, MMP-1 expression $(\mathrm{HR}=2.109,95 \% \mathrm{Cl}=$ 1.293-3.279), and PAR-1 expression ( $\mathrm{HR}=1.967,95 \% \mathrm{Cl}=1.256-2.881)$ as independent and significant prognostic factors for DFS. Our data suggest for the first time that MMP-1 and PAR-1 were both overexpressed in ESCC and are novel predictors of poor patient prognosis after curative resection. The MMP-1/PAR-1 signal transduction axis might be a new therapeutic target for future therapies tailored against ESCC.
\end{abstract}

Key words: Matrix metalloprotease-1; Protease-activated receptor-1; Esophageal squamous cell carcinoma; Prognosis; Immunohistochemistry

\section{Introduction}

Esophageal squamous cell carcinoma (ESCC), the major histological form of esophageal cancer in East Asian countries, is a highly aggressive malignancy with a poor prognosis (1). Because of its high frequency of lymph node metastases and recurrence, multimodality treatments including surgery, chemotherapy, and radiotherapy are often required to counter this disease. Despite such treatment combinations, the prognosis of patients with ESCC is still unsatisfactory (2). Assessment of prognosis based on clinicopathologic features remains inadequate even when using the staging systems of tumor node metastasis (TNM) classification because of the considerable variability and heterogeneity within the same stage (3). Therefore, it is important to evaluate and establish novel prognostic markers for ESCC to enable its diagnosis at an early stage when radical resection is possible.

The tumor metastatic cascade consists of multiple successive steps, including adhesion of tumor cells at primary site, invasion of intravascular space, dissemination to distant sites, adhesion of tumor cells to the vascular endothelium of distant tissues, extravasation and invasion of surrounding tissues, and finally formation of secondary tumor colonies $(4,5)$. To facilitate cell motility, invading cells need to change the cell-cell adhesion properties, rearrange the extracellular matrix (ECM) environment, suppress anoikis and reorganize their cytoskeletons (6). The degradation of the ECM during the migration process requires the action of proteolytic enzymes such as metalloproteinases (MMPs), which are zinc-dependent endopeptidases. Depending on the substrate specificity and structure, they are divided into several

Correspondence: Pei-guo Cao, Department of Oncology, the Third Xiangya Hospital, Central South University, Changsha, 410013, Hunan Province, China. Fax: +86-731-8529-5260. E-mail: pls0709@126.com

Received May 16, 2011. Accepted November 1, 2011. Available online November 18, 2011. Published January 16, 2012. 
subgroups: collagenases (e.g., MMP-1), stromelysins (e.g., MMP-3; MMP-10), matrilysins (e.g., MMP-7; MMP-26), gelatinases (e.g., MMP-2; MMP-9), and membrane-type MMP-1 (MT1-MMP) (7). It has been demonstrated that MMPs contribute to normal biological processes such as embryonic development and tissue repair, and also play a major role in the growth, invasion, and metastasis of malignant tumors (8). In particular, interstitial collagenase (MMP-1) is associated with poor patient prognosis in malignant melanoma and in breast, ovarian, colorectal, pancreatic, and gastric cancers (9-12). The findings of Grimm et al. (13) suggested that MMP-1 plays a role as a preinvasive factor in Barrett's esophagus-associated esophageal adenocarcinomas. They also found that the expression of MMP-1 in proliferating Barrett's esophagus and esophageal adenocarcinoma cells suggests malignant proliferation following the clonal expansion model (13). In addition, MMP-1 proteolytically activates the G-protein-coupled receptor protease-activated receptor-1 (PAR-1), suggesting that MMP-1 has a greater role in tumor progression by activating signal transduction pathways and modulating cell behavior. PAR-1 is also an oncogene, and is overexpressed in several types of cancers, including breast and colorectal cancers and melanoma (14-16). Signaling through PAR-1 facilitates tumor invasion, angiogenesis and metastasis by inducing the expression of genes associated with cell adhesion, invasion and survival.

However, the activation and the prognostic value of the MMP-1/PAR-1 signal transduction axis in ESCC are still uncertain. To further explore the expression patterns of MMP-1 and PAR-1 and to determine whether these proteins could be prognostic factors in ESCC, we investigated their expressions in a series of ESCC using immunohistochemical staining. The correlation of these proteins with survival of ESCC patients was also addressed.

\section{Patients and Methods}

\section{Patients and tissue samples}

The study was approved by the Research Ethics Committee of the Third Xiangya Hospital, the Central South University, China. Written informed consent was obtained from all patients. All specimens were handled and made anonymous according to ethical and legal standards.

The study involved 85 patients with histopathologically confirmed primary thoracic esophageal cancer who underwent surgical resection at our hospital between 2000 and 2005. They included 9 female and 76 male patients, ranging in age from 36 to 82 years (median 66.1 years). Subtotal esophagectomy via right thoracotomy with two- or three-field lymphadenectomy was performed in all patients. Curative resection (R0) was achieved in 81 patients (95.3\%), while the outcome for the remaining $4(4.7 \%)$ patients was noncurative resection (R1, R2). Forty-five patients with lymph node metastasis at initial diagnosis received neoadjuvant chemotherapy, which consisted of two courses of 5-fluorouracil (5-FU), cisplatin (CDDP), and adriamycin (ADM). We provided adjuvant chemotherapy (docetaxel or CDDP plus $5-\mathrm{FU}$ ) to 6 patients with larger numbers of pathologically positive lymph nodes. None of the patients died of postoperative complications.

After surgery, the patients were surveyed every 3 months by physical examination and serum tumor markers (squamous cell carcinoma antigen, carcinoembryonic antigen), every 6 months by computed tomography scan and abdominal ultrasonography, and every year by endoscopy until tumor recurrence was evident. Patients with tumor recurrence received chemotherapy or chemoradiotherapy, as long as their systemic condition permitted. Mean overall survival was 33.0 months and mean disease-free survival (DFS) was 26.8 months. The mean follow-up period after surgery was 42.6 months.

Clinical and pathologic parameters were obtained from the pathology reports and are presented in Table 1.

\section{Immunohistochemical analysis}

Tumor samples were fixed with $10 \%$ formaldehyde in phosphate-buffered saline (PBS), embedded in paraffin, and sectioned into 4- $\mu$ m thick sections. Immunohistochemical study for MMP-1 and PAR-1 was performed using the avidinbiotin-peroxidase complex method. Briefly, the sections were deparaffinized and dehydrated using a graded series of ethanol solutions. Endogenous peroxidase activity was inhibited by the administration of $0.3 \%$ hydrogen peroxidase and methanol for 20 min in each section. After rinsing in PBS, the tissue sections were processed in $0.01 \mathrm{M}$ citrate buffer, $\mathrm{pH} 6.0$, inside a heat-resistant plastic container. Sections were then irradiated in a domestic microwave oven for 20 min. After microwave irradiation, the slides were allowed to cool at room temperature. The following antibodies were applied as primary antibodies: mouse monoclonal anti-MMP-1 antibody (1:1000; sc-21731; Santa Cruz Biotechnology, USA), and goat polyclonal anti-PAR-1 antibody (1:1000; sc-8202; Santa Cruz Biotechnology). The sections were incubated with these primary antibodies overnight at $4^{\circ} \mathrm{C}$, followed by the anti-mouse (for MMP-1) and anti-goat (for PAR-1) secondary antibodies, respectively. The results were visualized with diaminobenzidine. In each immunohistochemistry experiment, sections of breast cancer were taken to be the positive control slides for both MMP-1 and PAR-1 staining. Negative controls were performed by replacing the primary antibodies with PBS.

MMP-1 and PAR-1 staining for each ESCC sample was judged to be positive when more than $10 \%$ (17) of the cancer cells in the section were immunoreactive to MMP-1 and PAR-1, respectively, and negative when $10 \%$ or less of the cells were positive to either marker. All slides were evaluated independently by two pathologists and then by conference in case of disagreement. Both pathologists were blind to the clinicopathologic data. 


\section{Statistical analysis}

The SPSS software version 12.0 for Windows (SPSS Inc., USA) and SAS 9.1 (SAS Institute, USA) was used for statistical analysis. The association of MMP-1 and PAR-1 expression with the clinicopathologic characteristics was analyzed using a contingency table. Statistical significance was evaluated with $x^{2}$ tests. Prognostic variables were assessed by the log-rank test, and free DFS was analyzed by the method of Kaplan and Meier. Cox's proportional hazard regression model with stepwise comparisons was used to analyze the independent prognostic factors. Differences were considered to be statistically significant when $\mathrm{P}$ was less than 0.05 .

\section{Results}

\section{Expression of MMP-1 and PAR-1 in ESCC}

A total of 85 samples (Table 1) that contained both cancerous and non-cancerous lesions were evaluated for MMP-1 and PAR-1 protein expression by immunohistochemical analysis. Of these, 62 (72.9\%) were positive for MMP-1 expression, mainly on the cell membranes and/or in the cytoplasm of tumor cells (Figure 1A), while the remaining $23(27.1 \%)$ were negative. PAR-1 immunoreactivity was observed in the cytoplasm of tumor cells in ESCC tissues (Figure 1C). Positive PAR-1 expression was observed in tumor cells of $68.2 \%(58 / 85)$ ESCC tissues. The positive staining was almost homogeneous in the single cancer nest and in different areas (surface, central, and deepest areas) of the cancer lesion. In contrast, none of the normal squamous epithelium showed a significant level of immunohistochemical staining for PAR-1 (Figure $1 \mathrm{~B}$ and $\mathrm{D})$. The grading of immunostained sections by the two pathologists was almost identical, with inter-observer variation of less than $10 \%$.

Association of MMP-1 and PAR-1 expression with clinicopathologic parameters. To obtain a better understanding of the clinical significance of MMP-1 and PAR-1 expression in ESCC, we correlated their expression with a series of clinicopathologic parameters. As shown in Table 1, MMP1 and PAR-1 overexpression was significantly associated with both TNM stage ( $P=0.021$ and 0.028 , respectively) and regional lymph node invasion $(P=0.012$ and 0.016 , respectively). There was no significant association with age, gender or histological grade (Table 1).

Correlation of MMP-1 and PAR-1 expression with

Table 1. Correlations of matrix metalloprotease-1 (MMP-1) and protease-activated receptor-1 (PAR-1) expression with the clinicopathological parameters of esophageal squamous cell carcinoma.

\begin{tabular}{|c|c|c|c|c|c|}
\hline \multirow[t]{2}{*}{ Clinicopathological parameters } & \multirow[t]{2}{*}{ No. of cases $(\%)$} & \multicolumn{2}{|c|}{ MMP-1 } & \multicolumn{2}{|c|}{ PAR-1 } \\
\hline & & Positive (\%) & Negative (\%) & Positive (\%) & Negative (\%) \\
\hline \multicolumn{6}{|l|}{ Age (years) } \\
\hline$<65$ & $37(43.5)$ & $26(70.3)$ & $11(29.7)$ & $25(67.6)$ & $12(32.4)$ \\
\hline$\geq 65$ & $48(56.5)$ & $36(75.0)$ & $12(25.0)^{*}$ & $33(68.8)$ & $15(31.2)^{*}$ \\
\hline \multicolumn{6}{|l|}{ Gender } \\
\hline Male & $76(89.4)$ & $56(73.7)$ & $20(26.3)$ & $52(68.4)$ & $24(31.6)$ \\
\hline Female & $9(10.6)$ & $6(66.7)$ & $3(33.3)^{*}$ & $6(66.7)$ & $3(33.3)^{*}$ \\
\hline \multicolumn{6}{|l|}{ Tumor location } \\
\hline Upper, middle & $49(57.6)$ & $36(73.5)$ & $13(26.5)$ & $32(65.3)$ & $17(34.7)$ \\
\hline Lower & $36(42.4)$ & $26(72.2)$ & $10(27.8)^{*}$ & $26(72.2)$ & $10(27.8)^{*}$ \\
\hline \multicolumn{6}{|l|}{ Histological differentiation grade } \\
\hline Well & $20(23.5)$ & $17(85.0)$ & $3(15.0)$ & $13(65.0)$ & $7(35.0)$ \\
\hline Moderate & $45(53.0)$ & $29(64.4)$ & $16(35.6)$ & $30(66.7)$ & $15(33.3)$ \\
\hline Poor & $20(23.5)$ & $16(80.0)$ & $4(20.0)^{*}$ & $15(75.0)$ & $5(25.0)^{*}$ \\
\hline \multicolumn{6}{|l|}{ Regional lymph node } \\
\hline No & $50(58.8)$ & $28(56.0)$ & $22(44.0)$ & $26(52.0)$ & $24(48.0)$ \\
\hline $\mathrm{N} 1$ & $35(41.2)$ & $34(97.1)$ & $1(2.9)^{*+}$ & $32(91.4)$ & $3(8.6)^{\star+}$ \\
\hline \multicolumn{6}{|l|}{ TNM stage } \\
\hline $\mathrm{l} / \mathrm{lla}$ & $49(57.6)$ & $29(59.2)$ & $20(40.8)$ & $27(55.1)$ & $22(44.9)$ \\
\hline Ilb/III & $36(42.4)$ & $33(91.7)$ & $3(8.3)^{\star+}$ & $31(86.1)$ & $5(13.9)^{\star+}$ \\
\hline
\end{tabular}

$\mathrm{N} 0=$ absence of regional lymph node metastasis; $\mathrm{N} 1$ = presence of regional lymph node metastasis; $\mathrm{TNM}=$ tumor node metastasis. ${ }^{*} \mathrm{P}<0.05$ compared to positive $\left(\mathrm{X}^{2}\right.$ test $) ;{ }^{+} \mathrm{P}<0.05$, significant difference between the variables of each parameter $\left(\mathrm{X}^{2}\right.$ test). 
survival. Disease recurrence after curative resection was diagnosed in $42(51.9 \%)$ of 81 patients and the mean time to recurrence was 9.6 months. The total 5-year DFS rate was $47.1 \%$. Patients with MMP-1and PAR-1-positive tumors, respectively, had poorer DFS than those with negative ESCC ( $P=0.002$ and 0.003 , respectively; Figure $2 \mathrm{~A}$ and $B$ ). Univariate analysis showed that the relationships between TNM stage [hazard ratio $(\mathrm{HR})=2.836$, $95 \%$ confidence interval $(\mathrm{Cl})=$ 1.866-4.308, $\mathrm{P}<0.0001$ ], regional lymph node involvement $(\mathrm{HR}=$ 2.955, $95 \% \mathrm{Cl}=1.713-5.068, \mathrm{P}<$ $0.0001), \mathrm{MMP}-1$ expression (HR = $2.669,95 \% \mathrm{Cl}=1.229-6.127, \mathrm{P}=$ 0.0070 ), and PAR-1 expression (HR $=1.762,95 \% \mathrm{Cl}=1.156-2.883, \mathrm{P}=$ 0.0072 ) and DFS were significant, but not between all other parameters tested (e.g., age, gender, histology grade, and tumor location; Table 2). Multivariate analysis using the above four parameters with statistical significance $(P<0.05)$ in univariate analysis identified TNM stage $(\mathrm{HR}=2.035,95 \% \mathrm{Cl}=$ 1.167-3.681, $\mathrm{P}=0.0120), \mathrm{MMP}-1$ expression $(\mathrm{HR}=2.109,95 \% \mathrm{Cl}=$ 1.293-3.279, $P=0.0008)$, and $P A R-$ 1 expression $(\mathrm{HR}=1.967,95 \% \mathrm{Cl}=$
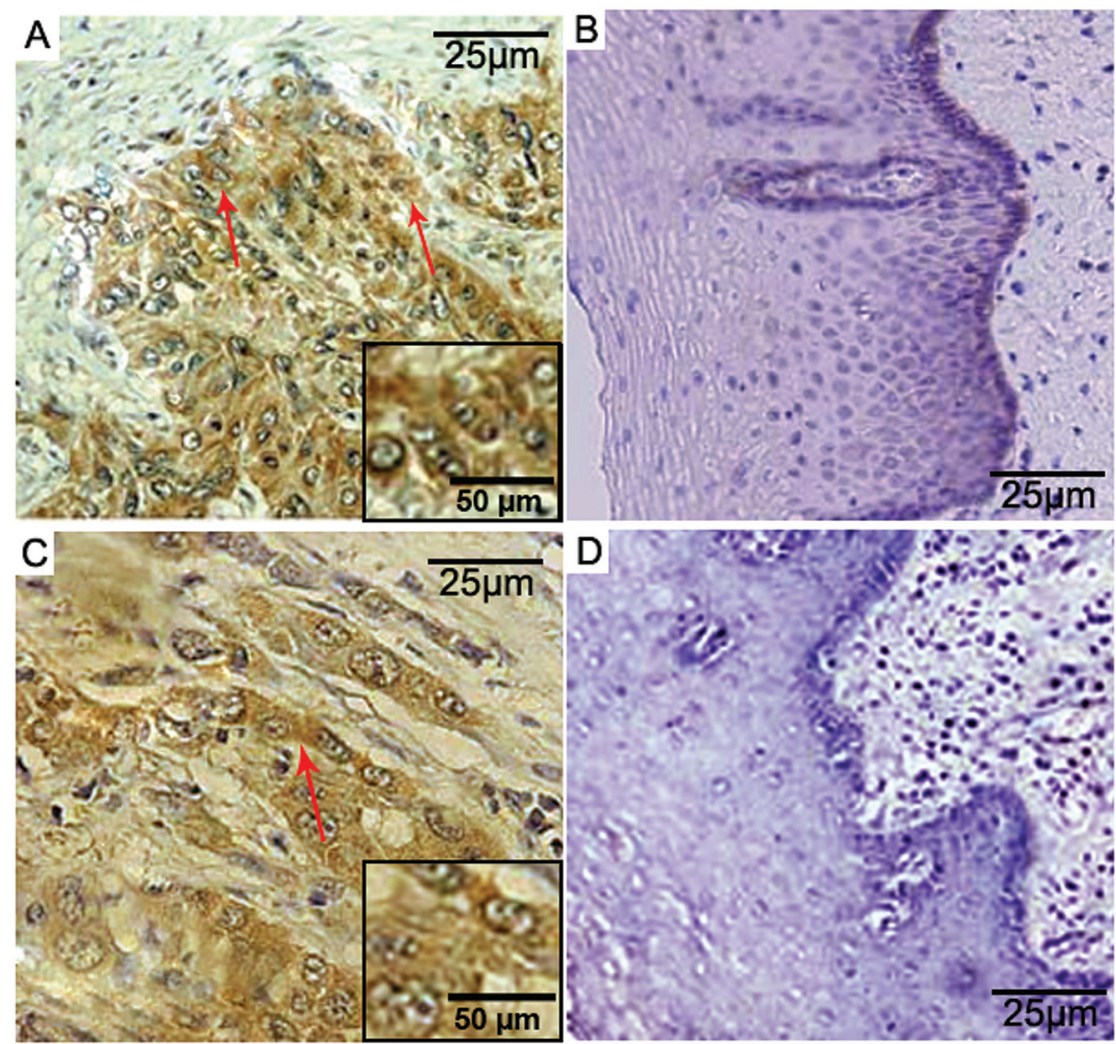

Figure 1. Matrix metalloprotease-1 (MMP-1) and protease-activated receptor-1 (PAR-1) expression determined by immunohistochemical staining. A, Representative MMP-1-positive esophageal squamous cell carcinoma showing staining mainly on the cell membranes and/ or in the cytoplasm of tumor cells (arrows); $B$, representative normal squamous epithelium negative for MMP-1 expression; $C$, representative PAR-1-positive esophageal squamous cell carcinoma showing staining mainly in the cytoplasm of tumor cells (arrow); $D$, representative normal squamous epithelium negative for PAR-1 expression.
A

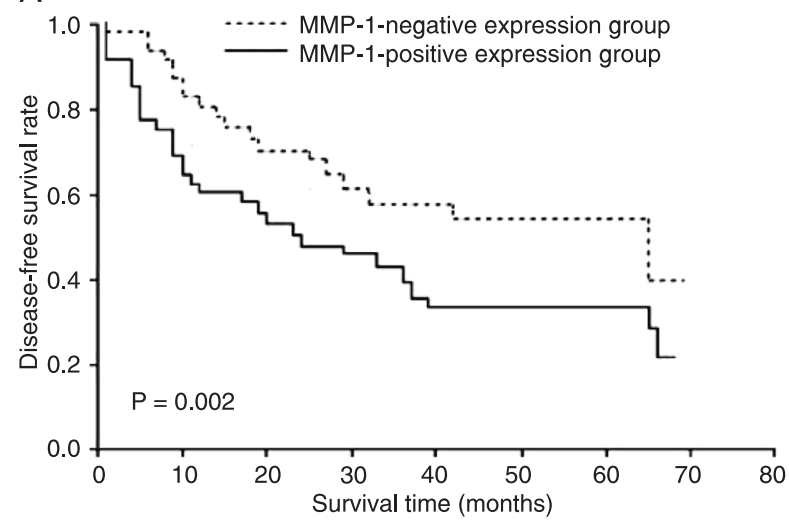

B

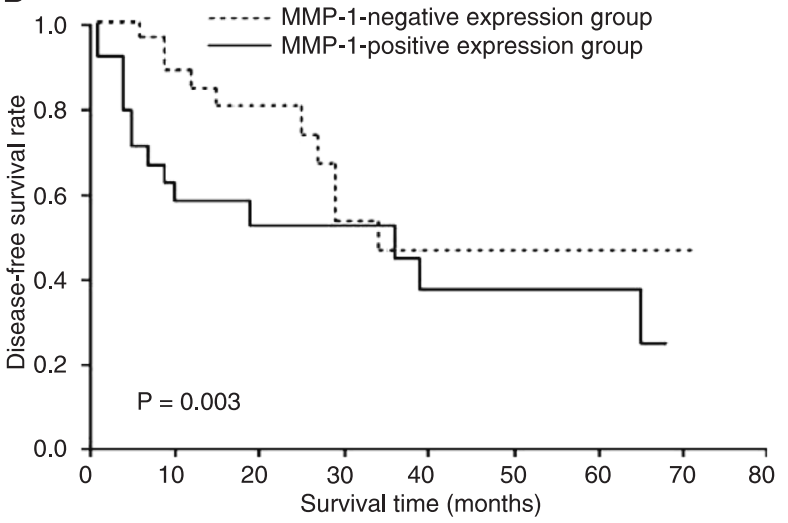

Figure 2. Survival curves according to matrix metalloprotease-1 (MMP-1) and protease-activated receptor-1 (PAR-1) expression. $A$, Disease-free survival curves according to MMP-1 expression for all patients plotted by the Kaplan-Meier method. $B$, Diseasefree survival curves according to PAR-1 expression for all patients plotted by the Kaplan-Meier method. Differences between the two groups were evaluated by the log-rank test. 
Table 2. Univariate analysis of disease-free survival by Cox's proportional hazard model.

\begin{tabular}{lcrr}
\hline Parameters & Hazard ratio & 95\% confidence interval & P \\
\hline Age (<65 years/ $\geq 65$ years) & 1.116 & $0.795-1.853$ & 0.5068 \\
Gender (male/female) & 1.018 & $0.660-1.629$ & 0.9521 \\
Tumor location (lower/upper-middle) & 1.521 & $0.996-2.324$ & 0.0833 \\
Histological differentiation grade (moderate-poor/well) & 1.016 & $0.510-2.033$ & 0.9326 \\
Regional lymph node (N0/N1) & 2.955 & $1.713-5.068$ & $<0.0001$ \\
TNM stage (I, lla/llb, III) & 2.836 & $1.866-4.308$ & $<0.0001$ \\
MMP-1 expression (positive/negative) & 2.669 & $1.229-6.127$ & 0.0070 \\
PAR-1 expression (positive/negative) & 1.762 & $1.156-2.883$ & 0.0072 \\
\hline
\end{tabular}

N0 = absence of regional lymph node metastasis; N1 = presence of regional lymph node metastasis; TNM = tumor node metastasis; MMP-1 = matrix metalloprotease-1; PAR-1 = protease-activated receptor -1 .

Table 3. Multivariate analysis of disease-free survival by Cox's proportional hazard model.

\begin{tabular}{lccc}
\hline Parameters & Hazard ratio & $95 \%$ confidence interval & $P$ \\
\hline Regional lymph node (N0/N1) & 2.055 & $0.913-4.658$ & 0.0680 \\
TNM stage (I, Ila/llb, III) & 2.035 & $1.167-3.681$ & 0.0120 \\
MMP-1 expression (positive/negative) & 2.109 & $1.293-3.279$ & 0.0008 \\
PAR-1 expression (positive/negative) & 1.967 & $1.256-2.881$ & 0.0009 \\
\hline
\end{tabular}

$\mathrm{N} 0=$ absence of regional lymph node metastasis; $\mathrm{N} 1$ = presence of regional lymph node metastasis; TNM = tumor node metastasis; MMP-1 = matrix metalloprotease-1; PAR-1 = protease-activated receptor- 1 .

1.256-2.881, $\mathrm{P}=0.0009$ ) as independent and significant prognostic factors for DFS (Table 3).

\section{Discussion}

The present study of MMP-1 and PAR-1 expression in 85 resected ESCC specimens showed positive tumor cell MMP-1 and PAR-1 expression in 72.9 and $68.2 \%$ of the specimens with negative immunostaining in the normal squamous epithelium, respectively. MMP-1 and PAR-1 protein expression correlated with TNM stage and regional lymph node invasion. With respect to prognosis, immunostaining of ESCC for MMP-1 and PAR-1 was a significant determinant of unfavorable prognosis. In addition to positive expression of MMP-1 and PAR-1, multivariate analysis also identified TNM stage as an independent prognostic predictor for DFS. Therefore, immunostaining for MMP-1 and PAR-1 could be considered for the prediction of an unfavorable prognosis for patients who undergo ESCC resection.

MMPs decompose the ECM and basal membrane and play a leading role in the process of tumor invasion and metastasis. Recent studies have demonstrated that MMPs are involved in early tumorigenesis by modulating cell proliferation, apoptosis, and host immune surveillance $(18,19)$. In human ESCC cells, MMPs have been shown to correlate with malignant grade and metastasis. For example, Yamashita et al. (20) studied 48 tumor/normal pair samples of human esophagus by Northern blot analysis and demonstrated that the tumor tissue of esophageal carcinoma showed a higher expression of MMP-7 mRNA than the corresponding normal tissue. Their findings also indicated that MMP-7 might be a novel prognostic factor for patients with esophageal carcinoma. Zhou et al. (21) demonstrated that the serum autoantibody levels of MMP-7 may be a good diagnostic biomarker for ESCC. In addition, Lukaszewicz-Zajac et al. (22) reported that serum levels of MMP-9 were significantly higher in ESCC patients compared to healthy subjects and serum concentrations of MMP-9 tended to increase in patients with advanced cancer. Mroczko et al. (23) also proved the synthesis of MMP-9 by cancer cells as well as by inflammatory cells and its correlation with tumor stage, tumor size, depth of tumor invasion, and lymph node metastases. Wu et al. (24) further showed that MMP-9 gene P574R polymorphism may contribute to a genetic risk factor for ESCC in a Chinese population. Moreover, the study of Shima et al. (25) suggested that MMP-2 and MMP-3 play an important role in tumor invasion and metastasis and that analysis of MMP-2 and MMP-3 production is useful for the evaluation of malignant potential in esophageal carcinomas. The results of Shen et al. (26) 
suggested that MT1-MMP is an enzyme responsible for Ln-5gamma2 cleavage in ESCC, and interaction between them may play a critical role in promoting invasion and metastasis of human ESCC. Furthermore, Ahokas et al. (27) reported that, during esophageal tumorigenesis, MMP-21 and MMP-26 have different, unique expression patterns both being tightly regulated and induced in the vicinity of inflammation. MMP-21 may provide a marker for differentiating tumor areas. Gu et al. (28) found that MMP-9 expression is a negative, independent prognostic factor in ESCC and correlates with tumor cell differentiation, vessel permeation, and lymph node metastasis. MMP-7, MMP9, and MMP-13 may function in early stage ESCC, and their co-expression predicts a poor outcome for relatively early stage ESCC patients. On the other hand, it has been reported that MMP plays an important role in thrombininduced metastasis in human cancer cells. Thrombin, the strongest activator of PAR-1, functions as a potent mesenchymal cell mitogen and chemoattractant, promoting the recruitment and proliferation of mesenchymal cells at sites of injury and in tumors. However, thrombin is not the only proteinase that activates PAR-1. Recent studies have revealed that PAR- 1 is activated by several additional proteinases (29). The function of PAR-1 is linked directly to the enzymatic activity of MMP-1. Among all MMPs, MMP-1 has the unique ability to cleave PAR-1, with subsequent activation of signal transduction pathways and alterations in the expression of downstream genes. This unique ability to cleave PAR-1 gives MMP-1 a powerful role in controlling cell behavior. Eck et al. (30) found that the MMP-1/PAR-1 signal transduction axis in endothelial cells activated mitogen-activated protein kinases, resulting in the induction of a panel of pro-angiogenic genes, while

\section{References}

1. Lv L, Pan K, Li XD, She KL, Zhao JJ, Wang W, et al. The accumulation and prognosis value of tumor infiltrating IL-17 producing cells in esophageal squamous cell carcinoma. PLoS One 2011; 6: e18219.

2. Yoon MS, Nam TK, Lee JS, Cho SH, Song JY, Ahn SJ, et al. VEGF as a predictor for response to definitive chemoradiotherapy and COX-2 as a prognosticator for survival in esophageal squamous cell carcinoma. J Korean Med Sci 2011; 26: 513-520.

3. Sakano A, Yanai H, Sakaguchi E, Tanioka Y, Yahara N, Hayashi $\mathrm{H}$, et al. Clinical impact of tumor invasion depth staging of esophageal squamous cell carcinoma using endoscopic ultrasonography. Hepatogastroenterology 2010; 57: 1423-1429.

4. Mehlen P, Puisieux A. Metastasis: a question of life or death. Nat Rev Cancer 2006; 6: 449-458.

5. Sternlicht MD, Werb Z. How matrix metalloproteinases regulate cell behavior. Annu Rev Cell Dev Biol 2001; 17: 463-516.

6. Pei D. Matrix metalloproteinases target protease-activated thrombin activated another panel of genes. Consequently, PAR-1 activation by MMP-1 vs thrombin could differentially influence cell behavior and pathological outcome. In addition, the two proteinases could cooperate in the tissue microenvironments to promote both tumor progression and inflammation. Since MMP-1 produced by activated fibroblasts in response to cytokines and growth factors is present in cancer, MMP-1 has the potential to activate PAR-1 on several types of cells, amplifying the role of fibroblast-derived MMP-1 as a collagenolytic enzyme and also as a critical signaling molecule. Blackburn et al. (31) further demonstrated that the MMP-1/PAR-1 signal transduction axis exists in vertical growth phase melanoma, and is necessary for melanoma invasion. Considered together, the above cited results and our findings suggest that MMP-1 and PAR-1 expression plays an important role in the development and/or progression of certain types of human tumors including ESCC.

We showed here that MMP-1 and PAR-1 were overexpressed in ESCC. MMP-1 and PAR-1 might be significant diagnostic markers for ESCC. More importantly, MMP-1 and PAR-1 could serve as independent prognostic markers for ESCC. Therefore, the MMP-1/PAR-1 signal transduction axis may play a significant role in the progression of ESCC, which is consistent with most other cancers. Further studies will be needed to establish the biological significance of MMP-1 and PAR-1 expression and to examine the possibility of their use as therapeutic targets in ESCC. We hope that the findings of this study will stimulate exploration of effective treatment strategies and the development of new therapeutic approaches for ESCC, such as antibody therapy or functional inhibition of expression.

receptors on the tumor cell surface. Cancer Cell 2005; 7: 207-208.

7. McCawley LJ, Crawford HC, King LE Jr, Mudgett J, Matrisian LM. A protective role for matrix metalloproteinase-3 in squamous cell carcinoma. Cancer Res 2004; 64: 69656972.

8. Poola I, DeWitty RL, Marshalleck JJ, Bhatnagar R, Abraham $\mathrm{J}$, Leffall LD. Identification of MMP-1 as a putative breast cancer predictive marker by global gene expression analysis. Nat Med 2005; 11: 481-483.

9. Alsaigh T, Pocock ES, Bergan JJ, Schmid-Schonbein GW. Acute venous occlusion enhances matrix metalloprotease activity: Implications on endothelial dysfunction. Microvasc Res 2011; 81: 108-116.

10. Hu F, Wang C, Guo S, Sun W, Mi D, Gao Y, et al. deltaEF1 promotes osteolytic metastasis of MDA-MB-231 breast cancer cells by regulating MMP-1 expression. Biochim Biophys Acta 2011; 1809: 200-210.

11. Wang FQ, Fisher J, Fishman DA. MMP-1-PAR1 axis mediates LPA-induced epithelial ovarian cancer (EOC) invasion. 
Gynecol Oncol 2011; 120: 247-255.

12. Chaudhary AK, Pandya S, Mehrotra R, Bharti AC, Jain S, Singh M. Functional polymorphism of the MMP-1 promoter $(-16071 \mathrm{G} / 2 \mathrm{G})$ in potentially malignant and malignant head and neck lesions in an Indian population. Biomarkers 2010; 15: 684-692.

13. Grimm M, Lazariotou M, Kircher S, Stuermer L, Reiber C, Hofelmayr A, et al. MMP-1 is a (pre-)invasive factor in Barrett-associated esophageal adenocarcinomas and is associated with positive lymph node status. $J$ Transl Med 2010; 8: 99.

14. Sze SC, Wong KL, Liu WK, Ng TB, Wong JH, Cheung HP, et al. Regulation of p21, MMP-1, and MDR-1 expression in human colon carcinoma HT29 cells by Tian Xian liquid, a Chinese medicinal formula, in vitro and in vivo. Integr Cancer Ther 2011; 10: 58-69.

15. Yin YJ, Salah Z, Maoz M, Ram SC, Ochayon S, Neufeld G, et al. Oncogenic transformation induces tumor angiogenesis: a role for PAR1 activation. FASEB J 2003; 17: 163-174.

16. Tellez C, Bar-Eli M. Role and regulation of the thrombin receptor (PAR-1) in human melanoma. Oncogene 2003; 22: 3130-3137.

17. Sano A, Kato H, Sakurai S, Sakai M, Tanaka N, Inose T, et al. CD24 expression is a novel prognostic factor in esophageal squamous cell carcinoma. Ann Surg Oncol 2009; 16: 506514.

18. Ho IA, Chan KY, Ng WH, Guo CM, Hui KM, Cheang P, et al. Matrix metalloproteinase 1 is necessary for the migration of human bone marrow-derived mesenchymal stem cells toward human glioma. Stem Cells 2009; 27: 1366-1375.

19. Agarwal A, Tressel SL, Kaimal R, Balla M, Lam FH, Covic $L$, et al. Identification of a metalloprotease-chemokine signaling system in the ovarian cancer microenvironment: implications for antiangiogenic therapy. Cancer Res 2010; 70: 5880-5890.

20. Yamashita K, Mori M, Shiraishi T, Shibuta K, Sugimachi K. Clinical significance of matrix metalloproteinase-7 expression in esophageal carcinoma. Clin Cancer Res 2000; 6: 1169-1174.

21. Zhou JH, Zhang B, Kernstine KH, Zhong L. Autoantibodies against MMP-7 as a novel diagnostic biomarker in esophageal squamous cell carcinoma. World J Gastroenterol 2011; 17: 1373-1378.

22. Lukaszewicz-Zajac M, Mroczko B, Kozlowski M, Niklinski
J, Laudanski J, Szmitkowski M. Elevated levels of serum metalloproteinase 9 in patients with esophageal squamous cell carcinoma. Pol Arch Med Wewn 2009; 119: 558-563.

23. Mroczko B, Kozlowski M, Groblewska M, Lukaszewicz M, Niklinski J, Laudanski J, et al. Expression of matrix metalloproteinase- 9 in the neoplastic and interstitial inflammatory infiltrate cells in the different histopathological types of esophageal cancer. Folia Histochem Cytobiol 2008; 46: 471-478.

24. Wu J, Zhang L, Luo H, Zhu Z, Zhang C, Hou Y. Association of matrix metalloproteinases-9 gene polymorphisms with genetic susceptibility to esophageal squamous cell carcinoma. DNA Cell Biol 2008; 27: 553-557.

25. Shima I, Sasaguri Y, Kusukawa J, Yamana H, Fujita H, Kakegawa T, et al. Production of matrix metalloproteinase-2 and metalloproteinase-3 related to malignant behavior of esophageal carcinoma. A clinicopathologic study. Cancer 1992; 70: 2747-2753.

26. Shen XM, Wu YP, Feng YB, Luo ML, Du XL, Zhang Y, et al. Interaction of MT1-MMP and laminin-5gamma2 chain correlates with metastasis and invasiveness in human esophageal squamous cell carcinoma. Clin Exp Metastasis 2007; 24: 541-550.

27. Ahokas K, Karjalainen-Lindsberg ML, Sihvo E, Isaka K, Salo $\mathrm{J}$, Saarialho-Kere U. Matrix metalloproteinases 21 and 26 are differentially expressed in esophageal squamous cell cancer. Tumour Biol 2006; 27: 133-141.

28. Gu ZD, Li JY, Li M, Gu J, Shi XT, Ke Y, et al. Matrix metalloproteinases expression correlates with survival in patients with esophageal squamous cell carcinoma. Am J Gastroenterol 2005; 100: 1835-1843.

29. Boire A, Covic L, Agarwal A, Jacques S, Sherifi S, Kuliopulos A. PAR1 is a matrix metalloprotease-1 receptor that promotes invasion and tumorigenesis of breast cancer cells. Cell 2005; 120: 303-313.

30. Eck SM, Blackburn JS, Schmucker AC, Burrage PS, Brinckerhoff $C E$. Matrix metalloproteinase and $G$ protein coupled receptors: co-conspirators in the pathogenesis of autoimmune disease and cancer. $J$ Autoimmun 2009; 33: 214-221.

31. Blackburn JS, Liu I, Coon Cl, Brinckerhoff CE. A matrix metalloproteinase-1/protease activated receptor-1 signaling axis promotes melanoma invasion and metastasis. Oncogene 2009; 28: 4237-4248. 